\title{
Experiência Inicial Utilizando a Via Radial no Tratamento Percutâneo de Doença Coronária
}

\author{
Clarissa Campo Dall'Orto', Guilherme Alves Lapa', Nádia de Mendonça Carnieto' ${ }^{1}$ Breno de Siqueira', \\ João Batista de Oliveira Neto', Maria Fernanda Zuliani Mauro', Salvador André Bavaresco Cristóvão', \\ Adnan Ali Salman'1, José Armando Mangione ${ }^{1}$
}

\section{RESUMO}

Introdução: A via radial é objeto de interesse crescente para procedimentos diagnósticos e terapêuticos, por possuir diversas vantagens, como comodidade para o paciente no pós-procedimento imediato, diminuição do tempo de internação com consequente redução dos custos hospitalares e menor índice de complicações no sítio de punção, quando comparada à via femoral. Método: Realizada análise retrospectiva de 2.027 pacientes consecutivos submetidos a intervenção coronária percutânea eletiva no período de junho de 2006 a janeiro de 2008. Comparamos os pacientes tratados pelas vias radial e femoral (grupos VR e VF, respectivamente) em relação a características clínicas, angiográficas e do procedimento, e evolução tanto hospitalar como tardia. Resultados: Angioplastia coronária por via radial foi realizada em $27 \%$ dos pacientes. O grupo VR mostrou maior número de pacientes do sexo masculino (75\% vs. $63,8 \%$; $\mathrm{P}<0,001)$ e com lesões uniarteriais $(45,1 \%$ vs. $37,3 \%$; $\mathrm{P}<0,001)$. Não encontramos diferença em relação a disfunção do ventrículo esquerdo, tipo de lesão tratada ou uso de inibidor da glicoproteína IIb/IIla. O sucesso angiográfico foi maior na via radial $(99 \%$ vs. $97,3 \% ; P=0,046)$. Houve menor número de eventos cardíacos adversos maiores com a via radial nas fases tanto hospitalar $(0,7 \%$ vs. $2 \%$; $\mathrm{P}=0,043)$ como tardia $(11,3 \%$ vs. $16,3 \%$; $\mathrm{P}<0,005)$, em virtude da menor complexidade clínica e angiográfica dos pacientes. Conclusão: A técnica radial, na curva inicial de aprendizado dos operadores e em pacientes selecionados, mostrou excelentes resultados clínicos quando comparada à femoral. Sua incorporação à prática clínica poderá ser uma opção adicional na abordagem de pacientes com perfil mais complexo.

Descritores: Artéria radial. Artéria femoral. Angioplastia transluminal percutânea coronária/métodos. Cateterismo cardíaco/métodos.

\footnotetext{
1 Hospital Beneficência Portuguesa - São Paulo, SP, Brasil.

Correspondência: José Armando Mangione. Rua Maestro Cardim, 769 -

1 SS - sala 71 - Bloco 1 - São Paulo, SP, Brasil - CEP 01323-900

E-mail: uci@uol.com.br

Recebido em: 20/3/2009 - Aceito em: 23/6/2009
}

\author{
ABSTRACT \\ Initial Experience of Percutaneous Treatment \\ of Coronary Lesions by Radial Access
}

Background: The radial access is object of increasing interest for diagnostic and therapeutic procedures, due to several advantages such as convenience for patients in the early post-procedure period, decreasing hospitalization time with a consequent reduction in hospital costs, and a lower puncture site complication rate when compared to the femoral approach. Methods: Retrospective analysis of 2,027 consecutive patients undergoing elective percutaneous transluminal coronary angioplasty (PTCA) from June, 2006 to January, 2008, comparing patients undergoing PTCA using radial and femoral access (RA group and FA group, respectively) in relation to clinical, angiographic and procedural characteristics, and early and late outcomes. Results: Radial access was used in $27 \%$ of the patients. The RA group included a larger number of males $(75 \%$ vs. $63.8 \% ; \mathrm{P}<0.001)$ with single vessel disease $(45.1 \%$ vs. $37.3 \% ; P<0.001)$. There were no differences for left ventricular dysfunction, type of lesion or use of Ilb/Illa glycoprotein inhibitor. Angiographic success was greater with radial access $(99 \%$ vs. 97.3\%; $\mathrm{P}=0.046)$. There were fewer major adverse cardiac events (MACE) with the use of radial access both during hospitalization $(0.7 \%$ vs. $2 \% ; \mathrm{P}=0.043)$ and the late follow-up $(11.3 \%$ vs. $16.3 \%$; $\mathrm{P}<0.005)$, due to the lower clinical and angiographic complexity of the patients. Conclusion: The radial technique, in the initial learning curve of operators and in selected patients, showed excellent clinical results when compared to the femoral technique. Its use in the clinical practice might be an additional option in patients with a more complex profile.

Descriptors: Radial artery. Femoral artery. Angioplasty, transluminal, percutaneous coronary/methods. Heart catheterization/methods. 
vantagens como: comodidade para o paciente no pósprocedimento imediato, com retorno precoce a suas atividades; diminuição do tempo de internação, com consequente redução dos custos hospitalares; e baixo índice de complicações do sítio de punção, comparativamente à via femoral, reduzindo a taxa de sangramento maior, que, por sua vez, está relacionada a aumento do risco de morte e eventos isquêmicos ${ }^{4-6}$.

Apesar dessas vantagens, a experiência com a via radial é limitada em grande parte dos centros, pois o procedimento requer curva de aprendizado mais longa que para a via femoral, utilizada na maioria dos casos.

A curva de aprendizado mais longa da via transradial deve-se ao fato de a artéria radial apresentar calibre menor e estar mais sujeita à ocorrência de espasmo que a via femoral, tornando difícil tanto sua punção como a manipulação dos cateteres. Ainda não está estabelecido, na literatura, qual o número necessário de procedimentos realizados para tornar-se competente na técnica ${ }^{7}$. A escolha do local de acesso vascular na maioria dos centros é mais uma questão de tradição, opinião e experiência do operador que uma decisão baseada em evidência.

O objetivo deste trabalho foi avaliar as características clínicas e angiográficas pré, per e pós-procedimento e a evolução hospitalar e tardia dos pacientes submetidos a angioplastia coronária por via radial comparada à via femoral.

\section{MÉTODO}

Realizamos um estudo retrospectivo, que incluiu pacientes submetidos a angioplastia coronária no período de junho de 2006 a janeiro de 2008. Foram realizadas 2.027 angioplastias coronárias eletivas nesse período, sendo $548(27 \%)$ por via radial, com 762 lesões tratadas, e 1.479 (73\%) por via femoral, com 2.205 lesões tratadas. Foram incluídos os portadores de doença arterial coronária assintomáticos, com prova isquêmica positiva, angina estável e também aqueles com síndrome coronária aguda sem elevação do segmento ST, podendo ser tratadas lesões de novo, reestenoses, assim como lesões em enxertos arteriais e venosos, além daqueles com disfunção ventricular esquerda. Foram excluídos apenas os pacientes com síndrome coronária aguda com elevação do segmento ST (angioplastia primária).

A seleção dos pacientes para a via radial foi feita avaliando-se inicialmente as condições vasculares locais, ficando sua utilização a critério do médico operador. O pulso radial era palpado, e então se realizava o teste de Allen, que busca avaliar a patência do arco palmar (perfusão da mão dentro de 10 segundos após a liberação da compressão da artéria ulnar).

A punção da artéria radial foi realizada no membro superior direito do paciente, estendido ao lado do corpo e com a palma da mão virada para cima, utilizando-se anestesia local realizada com $2 \mathrm{ml}$ a $3 \mathrm{ml}$ de lidocaína 2\%. Utilizamos, para puncionar a artéria, jelco 18 ou 20, pois, além de ser fino, tem paredes flexíveis, estando associado a menor incidência de formação de hematoma após primeira tentativa sem sucesso. Depois da punção, foram utilizadas bainhas arteriais específicas para a artéria radial (5 F a $7 \mathrm{~F}$ ), sendo administrados $10 \mathrm{mg}$ de mononitrato de isossorbida por via intra-arterial. Após o procedimento, a bainha arterial foi retirada em sala, sem reversão da heparina, sendo feito curativo compressivo com gaze e esparadrapo. O curativo compressivo foi retirado, em geral, duas a três horas após o procedimento.

A punção na via femoral é realizada após anestesia local com $15 \mathrm{ml}$ a $20 \mathrm{ml}$ de lidocaína $2 \%$, pela técnica de Seldinger, com agulha de punção 18 gauge, introduzindo-se bainhas arteriais com diâmetro de $5 \mathrm{~F}$ a $7 \mathrm{~F}$. Após o procedimento, a bainha arterial é retirada e o local da punção é comprimido manualmente por 20 minutos, sendo feito curativo compressivo também com gaze e esparadrapo. O paciente é mantido em repouso com o membro estendido, durante três a cinco horas, conforme o calibre da bainha arterial.

Nas duas técnicas, a heparinização é realizada de maneira habitual, na dose de $70 \mathrm{UI} / \mathrm{kg}$ a $100 \mathrm{UI} / \mathrm{kg}$ por via intracoronária. Todos os pacientes receberam ácido acetilsalicílico e clopidogrel, conforme as recomendações das diretrizes vigentes.

Comparamos as características clínicas e angiográficas pré-procedimento, as características do procedimento, e a evolução tanto hospitalar como tardia dos pacientes submetidos a angioplastia coronária eletiva pelas vias radial e femoral.

No acompanhamento clínico tardio, os pacientes foram avaliados por consulta médica ou por contato telefônico aos 30 dias, aos seis meses e um ano após a intervenção coronária percutânea. A partir de então, passaram a ser avaliados anualmente.

\section{Análise estatística}

A análise dos dados foi feita com o pacote estatístico SPSS (Statistical Package for Social Sciences) e R ( $R$ Development Core Team) versão 2.6.0. Todos os testes foram realizados considerando hipóteses bilaterais e considerando um nível de significância $\alpha=5 \%$. Inicialmente foi utilizada a estatística descritiva para avaliar a frequência, a média e o desvio padrão das variáveis de interesse. Os dados quantitativos foram apresentados na forma de média \pm desvio padrão. As comparações das variáveis contínuas que não apresentaram distribuição normal foram realizadas por meio do teste de Mann-Whitney. As variáveis categóricas foram analisadas com o teste de qui-quadrado ou exato de Fisher, quando apropriado. 
A regressão logística múltipla com o método de seleção stepwise foi aplicada para encontrar possíveis preditores para os desfechos eventos adversos cardíacos maiores hospitalares e tardios.

\section{RESULTADOS}

Com relação às características clínicas e demográficas (Tabela 1), o grupo via radial mostrou maior número de pacientes do sexo masculino (75\% vs. $63,8 \% ; P<0,001)$ e tabagistas $(23,9 \%$ vs. $19,3 \%$; $\mathrm{P}=0,022)$ e menor prevalência de dislipidemia $(60,6 \%$ vs. $65,7 \% ; P=0,034)$ e cirurgia de revascularização do miocárdio prévia (9,3\% vs. 19,8\%; P < 0,001).

$\mathrm{Na}$ análise das características angiográficas e do procedimento (Tabela 2), houve diferença no número de vasos acometidos, com os pacientes com lesão uniarterial predominando no grupo da via radial e os triarteriais no grupo da via femoral $(45,1 \%$ vs. $37,3 \%$ e $19,3 \%$ vs. $27,8 \%$, respectivamente; $\mathrm{P}<0,001$ ), e em relação ao vaso tratado, com predominância de enxertos de ponte de veia safena e arteriais na via femoral e de artérias coronárias nativas na via radial. Não houve diferença em relação à disfunção grave do ventrículo esquerdo, ao tipo de lesão segundo a classificação do American College of Cardiology/American Heart Association e ao uso de inibidor da glicoproteína Ilb/ IIla. A necessidade de troca de via de acesso por falha de punção ocorreu somente na via radial (3\% vs. 0; $\mathrm{P}<0,001)$. O sucesso angiográfico foi maior na via radial (99\% vs. 97,3\%; $\mathrm{P}=0,046)$.

Na evolução hospitalar (Tabela 3), não foi registrada diferença nas taxas de óbito, infarto agudo do miocárdio ou necessidade de revascularização urgen-

TABELA 1

Características clínicas e demográficas

\begin{tabular}{lccc}
\hline & Via radial $(\mathbf{n}=\mathbf{5 4 8})$ & Via femoral (n= 1.479) & P \\
\cline { 1 - 3 } Idade, anos & $62,65 \pm 11,04$ & $63,59 \pm 11,43$ & 0,112 \\
Sexo feminino & $137(25 \%)$ & $535(36,2 \%)$ & $<0,001$ \\
Dislipidemia & $332(60,6 \%)$ & $971(65,7 \%)$ & 0,034 \\
Hipertensão arterial & $430(78,5 \%)$ & $1.186(80,2 \%)$ & 0,392 \\
Diabetes melito & $140(25,5 \%)$ & $398(26,9 \%)$ & 0,537 \\
Tabagismo & $131(23,9 \%)$ & $285(19,3 \%)$ & 0,022 \\
Revascularização miocárdica prévia & $51(9,3 \%)$ & $293(19,8 \%)$ & 0,001 \\
Quadro clínico & $75(13,8 \%)$ & $195(13,3 \%)$ & 0,456 \\
$\quad$ Assintomático & $292(53,8 \%)$ & $830(56,1 \%)$ & $436(29,4 \%)$ \\
$\quad$ Angina estável & $176(32,4 \%)$ & & \\
$\quad$ Angina instável & & & \\
\hline $\mathrm{n}=$ número de pacientes. & & & \\
\hline
\end{tabular}

TABELA 2

Características angiográficas e do procedimento

\begin{tabular}{|c|c|c|c|}
\hline & Via radial $(n=548)$ & Via femoral $(n=1.479)$ & $\mathbf{P}$ \\
\hline Tipo de lesão (ACC/AHA) & & & 0,259 \\
\hline$A+B 1$ & $326(42,8 \%)$ & $899(40,8 \%)$ & \\
\hline B2 & $213(28 \%)$ & $595(27 \%)$ & \\
\hline $\mathrm{C}$ & $223(29,3 \%)$ & $711(32,2 \%)$ & \\
\hline Número de vasos acometidos & & & $<0,001$ \\
\hline Uniarteriais & $247(45,1 \%)$ & $552(37,3 \%)$ & \\
\hline Biarteriais & $194(35,4 \%)$ & $484(32,7 \%)$ & \\
\hline Triarteriais & $106(19,3 \%)$ & $412(27,8 \%)$ & \\
\hline Disfunção grave do ventrículo esquerdo & $15(2,7 \%)$ & $50(3,4 \%)$ & 0,564 \\
\hline Uso do inibidor da glicoproteína Ilb/Illa & $15(2,7 \%)$ & $41(2,8 \%)$ & 0,966 \\
\hline Crossover de via de acesso & $16(3 \%)$ & - & $<0,001$ \\
\hline Sucesso angiográfico & $542(99 \%)$ & $1.439(97,3 \%)$ & 0,046 \\
\hline
\end{tabular}


te, havendo, no entanto, diferença na taxa combinada de eventos cardíacos adversos maiores nos pacientes tratados por via femoral $(0,7 \%$ vs. $2 \% ; \mathrm{P}=0,043)$. $\mathrm{O}$ sucesso clínico foi maior na via radial (98\% vs. 95,3\%; $\mathrm{P}=0,008)$.

Quando aplicamos o modelo de regressão logística (Tabela 4), o risco de eventos cardíacos adversos maiores na fase hospitalar nos pacientes submetidos à angioplastia coronária por via femoral foi 2,94 vezes maior comparativamente à via radial. Não foi possível aplicar esse método estatístico para os desfechos óbito, infarto agudo do miocárdio e necessidade de revascularização urgente em virtude do pequeno número desses eventos na população estudada.

No seguimento clínico tardio (Tabela 5), 94,7\% dos pacientes foram acompanhados por um período médio de $328 \pm 250,71$ dias. Pacientes tratados por via femoral mostraram maiores taxas de eventos cardíacos adversos maiores (11,3\% vs. 16,3\%; P<0,005), em decorrência da maior necessidade de nova revascularização (8,4\% vs. $12 \% ; P=0,02)$.

$\mathrm{Na}$ regressão logística (Tabela 6), os pacientes tratados por via femoral apresentaram risco $1,48 \mathrm{vez}$ maior de eventos cardíacos adversos maiores no seguimento tardio, bem como os pacientes com acometimento biarterial ou triarterial (risco 1,46 e 1,78 vez maior, respectivamente).

\section{DISCUSSÃO}

A técnica radial apresenta inúmeras vantagens: 1) ausência de grandes nervos ou veias adjacentes à artéria radial, tornando baixo o risco de lesão nervosa ou fístula arteriovenosa ${ }^{8,9}$; 2) maior conforto para o paciente, pois permite a deambulação imediata; 3) redução dos cuidados de enfermagem com a via de acesso; e 4) alta hospitalar precoce, com redução de custos. Além disso, apresenta menor taxa de complicações vasculares, tornando-se opção preferencial no grupo de pacientes de alto risco, como obesos, pacientes com doença aorto-ilíaca, em tratamento com anticoagulante ou inibidores da glicoproteína Ilb/IIla ou aqueles na fase aguda do infarto tratados com trombolíticos.

Diferenças no perfil de pacientes encontrados em nosso estudo, como maior prevalência do sexo feminino ou de pacientes com cirurgia de revascularização

TABELA 3

Evolução hospitalar

\begin{tabular}{lccc}
\hline & Via radial $(\mathbf{n}=\mathbf{5 4 8})$ & Via femoral $(\mathbf{n}=\mathbf{1 . 4 7 9 )}$ & $\mathbf{P}$ \\
\hline Sucesso clínico & $537(98 \%)$ & $1.409(95,3 \%)$ & 0,008 \\
ECAM & $4(0,7 \%)$ & $30(2 \%)$ & 0,043 \\
Óbito & $2(0,4 \%)$ & $13(0,9 \%)$ & 0,230 \\
Infarto agudo do miocárdio & $2(0,4 \%)$ & $15(1 \%)$ & 0,182 \\
RVA urgente & - & $2(0,1 \%)$ & $>0,99$ \\
Pseudoaneurisma & - & $18(1,2 \%)$ & 0,02
\end{tabular}

ECAM = eventos cardíacos adversos maiores; $\mathrm{n}$ = número de pacientes; RVA = revascularização do vaso-alvo.

TABELA 4

Regressão logística considerando ECAM hospitalar como variável dependente

\begin{tabular}{|c|c|c|c|}
\hline Variáveis & & OR & IC 95\% \\
\hline \multirow[t]{2}{*}{ Acesso } & Radial & - & - \\
\hline & Femoral & 2,94 & $1,01-8,53$ \\
\hline \multirow[t]{3}{*}{ DAC } & Uniarterial & - & - \\
\hline & Biarterial & 0,53 & $0,20-1,40$ \\
\hline & Triarterial & 1,98 & $0,84-4,68$ \\
\hline \multirow[t]{2}{*}{ Tabagismo } & Não & - & - \\
\hline & Sim & 2,12 & $0,97-4,61$ \\
\hline \multirow[t]{2}{*}{ Gênero } & Masculino & - & - \\
\hline & Feminino & 1,22 & $0,58-2,55$ \\
\hline
\end{tabular}


TABELA 5

Seguimento clínico tardio

\begin{tabular}{lccc}
\hline & Via radial $(\mathbf{n}=\mathbf{5 4 8})$ & Via femoral $(\mathbf{n}=\mathbf{1 . 4 7 9 )}$ & $\mathbf{P}$ \\
\hline Pacientes seguidos & $498(91 \%)$ & $1.360(91,9 \%)$ & 0,497 \\
Tempo de seguimento, dias & $320 \pm 266,19$ & $336 \pm 235,23$ & 0,190 \\
ECAM & $62(11,3 \%)$ & $241(16,3 \%)$ & 0,005 \\
Óbito & $11(2 \%)$ & $56(3,8 \%)$ & 0,047 \\
Infarto agudo do miocárdio & $6(1,1 \%)$ & $32(2,2 \%)$ & 0,115 \\
RVA & $46(8,4 \%)$ & $178(12 \%)$ & 0,02 \\
\hline
\end{tabular}

ECAM = eventos cardíacos adversos maiores; $\mathrm{n}$ = número de pacientes; RVA = revascularização do vaso-alvo.

TABELA 6

Regressão logística considerando ECAM tardio como variável dependente

\begin{tabular}{llcc}
\hline Variáveis & & OR & IC 95\% \\
\hline Acesso & Radial & - & - \\
& Femoral & 1,48 & $1,09-2,00$ \\
DAC & Uniarterial & - & - \\
& Biarterial & 1,46 & $1,08-1,98$ \\
& Triarterial & 1,78 & $1,25-2,54$ \\
Tabagismo & Não & - & - \\
\multirow{2}{*}{ Gênero } & Sim & 1,00 & $0,73-1,36$ \\
& Masculino & - & $0,65-1,12$ \\
\hline DAC = doença arterial coronária; ECAM = eventos cardíacos adversos maiores; IC = intervalo de confiança; OR = odds ratio. \\
\hline
\end{tabular}

do miocárdio prévia, abordados preferencialmente pela via femoral, podem ser justificadas pela curva de aprendizado. As mulheres apresentam, em geral, artérias mais finas e com maior chance de espasmo, fato que dificulta o procedimento na fase inicial do emprego dessa via de acesso.

Encontramos maiores taxas de insucesso e de eventos cardíacos adversos maiores, tanto na fase hospitalar como tardia, nos pacientes tratados por via femoral, o que pode ser justificado pela maior complexidade e extensão da doença coronária nesses pacientes.

A via radial, em nossa experiência, mostrou-se segura e eficaz, sendo dependente da curva de aprendizado do operador, com índice de sucesso equivalente ao da via femoral, com a vantagem de apresentar menores taxas de complicações vasculares, à semeIhança dos resultados do estudo ACCESS ${ }^{10}$. Nesse estudo, a via radial foi comparada à braquial e à femoral e mostrou menores taxas de sangramento, eventos isquêmicos e óbito ${ }^{4,5}$.

Labrunie et al. ${ }^{11}$ demonstraram recentemente a eficácia da via radial quando comparada às vias clássicas (Sones e via femoral) na realização de coronario- grafia, com índice de sucesso e complicações similares, mesmo durante aprendizagem.

\section{Limitações do estudo}

Por se tratar de um estudo observacional, temos todas as limitações inerentes a esse tipo de estudo. Não houve randomização dos pacientes que seriam selecionados para as abordagens radial e femoral, e isso era decidido avaliando-se as condições vasculares locais e levando-se em consideração a anatomia coronária, como já dito anteriormente.

Não foi calculado o tempo de fluoroscopia, que é um medidor da complexidade do procedimento por via radial, e que na literatura é maior que na via femoral ${ }^{11,12}$. Também não foi avaliada a duração da permanência hospitalar, não foi feita análise de custos, e tampouco foi avaliada e quantificada a presença de sangramentos relacionados ao sítio de punção.

\section{CONCLUSÃO}

Este estudo nos permite concluir que, em pacientes selecionados, a técnica radial é segura para proce- 
dimentos coronários com resultados clínicos similares aos da abordagem femoral. Além disso, virtualmente elimina complicações vasculares e pode diminuir os custos hospitalares por proporcionar alta precoce. Entretanto, é necessário que seja alcançada proficiência na técnica, pois a via radial é tecnicamente mais desafiadora que a via femoral.

\section{CONFLITO DE INTERESSES}

Os autores declararam inexistência de conflito de interesses.

\section{REFERÊNCIAS BIBLIOGRÁFICAS}

1. Campeau L. Percutaneous radial artery approach for coronary angiography. Cathet Cardiovasc Diagn. 1989;16(1):3-7.

2. Kiemeneij F, Laarman GJ, Melker E. Transradial artery coronary angioplasty. Am Heart J. 1995;129(1):1-7.

3. Kiemeneij F, Laarman GJ, Melker E. Transradial artery PalmazSchatz coronary stent implantation: results of a single-center feasibility study. Am Heart J. 1995;130(1):14-21.

4. Eikelboom JW, Mehta SR, Anand SS, Xie C, Fox KA, Yusuf S. Adverse impact of bleeding on prognosis in patients with acute coronary syndromes. Circulation. 2006;114(8):774-82.

5. Rao SV, O'Grady K, Pieper KS, Granger CB, Newby LK, Van de Werf $F$, et al. Impact of bleeding severity on clinical outcomes among patients with acute coronary syndromes. Am J Cardiol. 2005;96(9):1200-6.
6. Jolly SS, Amlani S, Hamon M, Yusuf S, Mehta SR. Radial versus femoral access for coronary angiography or intervention and the impact on major bleeding and ischemic events: a systematic review and meta-analysis of randomized trials. Am Heart J. 2009;157(1):132-40.

7. Nunes GL, Oliveira AT, Alves L, Alfonso T. Influência da curva de aprendizado no sucesso e na ocorrência de complicações associadas aos procedimentos pela via radial. Rev Bras Cardiol Invas. 2007;15(2):115-8.

8. Valsecchi O, Vassileva A, Musumeci G, Rossini R, Tespili M, Guagliumi G, et al. Failure of transradial approach during coronay interventions: anatomic considerations. Catheter Cardiovasc Interv. 2006;67(6):870-8.

9. Rihal CS, Holmes DR Jr. Transradial cardiac catheterization: is femoral access obsolete? Am Heart J. 1999;138(3 Pt 1): 392-3.

10. Kiemeneij F, Laarman GJ, Odekerken D, Slagboom T, van der Wieken R. A randomized comparison of percutaneous transluminal coronary angioplasty by the radial, brachial and femoral approaches: the ACCESS study. J Am Coll Cardiol. 1997;29(6): 1269-75.

11. Labrunie A, Tebet MA, Andrade PB, Andrade MVA, Conterno LO, Mattos LA, et al. Coronariografia via transradial: curva de aprendizagem, avaliada por estudo multicêntrico. Rev Bras Cardiol Invas. 2009;17(1):82-7.

12. Agostoni P, Biondi-Zoccai GG, de Benedictis ML, Rigattieri $S$, Turri M, Anselmi M, et al. Radial versus femoral approach for percutaneous coronary diagnostic and interventional procedures: systematic overview and meta-analysis of randomized trials. J Am Coll Cardiol. 2004;44(2):349-56. 